\title{
Health-care utilization for headache disorders in Nepal: a population-based door-to-door survey
}

\author{
Kedar Manandhar ${ }^{1,2}$, Ajay Risal $^{1,2}$, Mattias Linde ${ }^{3,4}$ and Timothy J. Steiner ${ }^{3,5^{*}}$
}

\begin{abstract}
Background: Headache disorders are an important global public-health problem, but under-diagnosed, undertreated and under-prioritized. Deficiencies in health care for headache, present everywhere, are likely to be greater in poorly-resourced countries. This study reports on health-care utilization for headache in Nepal, a lowincome country with high headache burden.
\end{abstract}

Methods: We took data from a cross-sectional, nationwide population-based door-to-door survey, with multistage cluster random sampling. Face-to-face structured interviews included enquiry into consultations with professional health-care providers (HCPs), and investigations and treatments for headache. Analysis included associations with sociodemographic variables and indices of symptom severity.

Results: Of 2100 participants, 1794 reported headache during the preceding year (mean age $36.1 \pm 12.6$ years; male/female ratio 1:1.6). Of these, $58.4 \%$ (95\% Cl: 56.1-60.7\%) had consulted at least once in the year with HCPs at any level, most commonly (25.0\%) paramedical; $15.0 \%$ had consulted pharmacists, $10.8 \%$ general physicians and $7.6 \%$ specialists (of any type). Participants with probable medication-overuse headache consulted most (87.0\%), followed by those with migraine $(67.2 \%)$ and those with tension-type headache (48.6\%; $p<0.001)$. A minority (11.9\%) were investigated, mostly (8.9\%) by eye tests. Half (50.8\%) had used conventional medications for headache in the preceding month, paracetamol being by far the most common (38.0\%), and 10.3\% had used herbal therapies. Consultation was positively associated with rural habitation ( $A O R=1.5 ; p<0.001$ ). Proportions consulting increased in line with all indices of symptom severity.

Conclusions: Although over half of participants with headache had consulted professional HCPs, this reflects demand, not quality of care. Although $7.6 \%$ had seen specialists, very few would have been headache specialists in any sense of this term. High persistent burden, with only half of participants with headache using conventional medications, and these not best chosen, suggests these consultations fell far short of meeting need. Health policy in Nepal should recognise this, since the consequences otherwise are costly: lost health, diminished productivity and damaged national economy. On a positive note, the proportions consulting suggest that capacity exists at multiple levels within the Nepalese health system. With this to build upon, structured headache services in line with international recommendations appear achievable in Nepal. Educational programmes are the essential requirement.

Keywords: Headache disorders, Health-care utilization, Medical consultation, Population-based study, Quality of care, Structured headache services, Nepal, Global campaign against headache

\footnotetext{
* Correspondence: t.steiner@imperial.ac.uk

${ }^{3}$ Department of Neuromedicine and Movement Science, Norwegian

University of Science and Technology, Edvard Griegs Gate, NO-7489

Trondheim, Norway

${ }^{5}$ Division of Brain Sciences, Imperial College London, London, UK

Full list of author information is available at the end of the article
}

(c) The Author(s). 2018 Open Access This article is distributed under the terms of the Creative Commons Attribution 4.0 International License (http://creativecommons.org/licenses/by/4.0/), which permits unrestricted use, distribution, and reproduction in any medium, provided you give appropriate credit to the original author(s) and the source, provide a link to the Creative Commons license, and indicate if changes were made. 


\section{Background}

Headache disorders are among the most prevalent, burdensome and costly diseases in the world [1-3]. From a public-health perspective, the primary headache disorders, mostly migraine and tension-type headache (TTH), are of special importance because they lead to widespread ill health, impaired quality of life and much loss of productivity [3]. Inappropriate management of either migraine or TTH can lead to medication-overuse headache (MOH), a major additional contributor to global disability [4].

The Global Burden of Disease Study 2015 (GBD2015) ranked TTH and migraine as second and fifth most prevalent disorders worldwide [5]. In GBD2016, migraine was the second highest cause of disability [6]. These disorders are not the preserve of wealthy countries: recent epidemiological studies in low- and middle-income (LAMI) countries (Ethiopia [7, 8], India [9, 10], Pakistan [11] and Zambia [12, 13]) all found higher prevalence estimates of migraine than the global mean, with substantial headache-attributed burden at individual and population levels. Despite these findings, headache disorders are under diagnosed, undertreated and under-prioritized in health-care delivery systems, and this is especially so in LAMI countries [1].

Nepal is one of the poorest countries within the South-East Asia Region (SEAR). Of its population of approximately 30 million, about one quarter live below the international poverty line [14]. In a population-based study in this country, we found an exceptionally high 1 -year prevalence of migraine $(34.1 \%)$, while the prevalence of probable $\mathrm{MOH}(\mathrm{pMOH})(2.1 \%)$ was towards the upper end of the range observed in other countries [15]. Headache-attributed burden was accordingly high: migraine, $\mathrm{TTH}$ and $\mathrm{pMOH}$ were accountable for reduced functional capacities of $0.81 \%, 0.06 \%$ and $0.20 \%$ respectively at population level [16].

Extreme geographical variation in Nepal causes difficulties of access, aggravated by poor infrastructure such as bad roads and rickety bridges, and a monsoon climate [17], while limited education generally, lack of skill in headache disorders among health professionals, and low investment in health care are high barriers to effective headache care in Nepal. This study assesses health-care utilization for headache and its associations with sociodemographic variables and indices of symptom severity in Nepal using data from the same nationwide population-based survey. The purpose was to provide evidence for national health policy and formulation of public-health programmes in Nepal.

\section{Methods}

\section{Study design}

The detailed methods have been described elsewhere [17, 18]. In summary, this was a cross-sectional, nationwide population-based door-to-door survey. Trained interviewers made unannounced visits to households selected through stratified multistage cluster random sampling, and conducted face-to-face structured interviews with one adult randomly selected from each household. Representativeness was achieved by sampling in all three physiographic divisions (Mountain, Hill and Terai), and, within each division, all five development regions (Far-western, Mid-western, Western, Central and Eastern). In total, 2100 Nepali-speaking adults aged 18-65 years and resident in Nepal were included during May 2013.

\section{Instrument}

Interviewers used the Headache-Attributed Restriction, Disability, Social Handicap and Impaired Participation (HARDSHIP) modular structured questionnaire developed by Lifting The Burden (LTB) for population-based studies [19]. This was culturally adapted, and translated into Nepali language according to LTB's translation protocol for hybrid documents [20]. It included multiple elements. First were (i) demographic enquiry and (ii) a neutral headache screening question ("Have you had a headache during the last 12 months?") addressed to all participants. Then, for those who screened positively, were (iii) diagnostic questions based on the International Classification of Headache Disorders (ICHD) [21] (focused on the subjectively most bothersome type in those reporting more than one), and (iv) questions regarding various aspects of headache-attributed burden. The last included indices of symptom severity: headache frequency (days/month $[\mathrm{d} / \mathrm{m}]$ ), attack duration (hours) and headache intensity (with response options "not bad", "quite bad" and "very bad", which we interpreted as mild, moderate and severe).

Last, of those with headache, we enquired about (v) consultations for headache (yes or no, and with whom: see below), (vi) investigations for headache undergone within the preceding year (particularly specifying xrays of paranasal sinuses [PNS] or neck, brain imaging [CT or MRI], EEG and eye tests), and (vii) treatments for headache (conventional medications and/or herbal preparations) in the preceding month.

\section{Headache diagnosis}

The diagnostic method, centrally by algorithm, has been described previously [15]. Participants reporting headache on $\geq 15 \mathrm{~d} / \mathrm{m}$ were first separated; those also overusing acute medication were considered to have $\mathrm{pMOH}$ [22] while the remainder were categorized as "other headache on $\geq 15 \mathrm{~d} / \mathrm{m}$ " (these cannot be fully diagnosed by questionnaire). To all others, reporting headache on $\leq 14 \mathrm{~d} / \mathrm{m}$, the algorithm applied modified ICHD-3 beta criteria [21] in the following order: definite migraine, definite TTH, probable migraine and probable TTH. 
Definite and probable migraine were combined, and likewise definite and probable TTH, for further analyses. The few remaining cases were unclassifiable.

\section{Medical consultation}

We defined "medical consultation" operationally as consultation, at least once in the preceding year, with any professional health-care provider (HCP) by a participant reporting any type of headache. We classified HCPs into four groups: (i) pharmacist; (ii) paramedic (nurse, physiotherapist, health assistant, auxiliary health worker or herbal practitioner); (iii) general physician; or (iv) specialist (headache specialist, neurologist, neurosurgeon, ophthalmologist, ear, nose and throat [ENT] specialist or psychiatrist). We ranked these groups in ascending order, (i) to (iv).

\section{Statistical analyses}

We counted participants with headache reporting medical consultation(s), investigations and/or treatments, and calculated proportions (\%) with 95\% confidence intervals (CIs). Those reporting consultations at multiple levels were counted only within the highest-ranked group.

We analysed demographic variables as follows. We categorized age in years into five groups: $18-25,26-35$, 36-45, 46-55, 56-65. We used household consumption in USD/year (at the time of study: 1 USD $\approx N P R \quad 100$ ) as an indicator of economic wellbeing, categorizing it in three groups: poorest $(<950)$, poor $(950-1200)$ and intermediate and above $(>1200)$. We classed habitation as urban or rural, and dichotomized altitude of dwelling into $<1000 \mathrm{~m}$ and $\geq 1000 \mathrm{~m}$.

As indices of symptom severity, we categorized headache frequency in $\mathrm{d} / \mathrm{m}(\mathrm{F})$ into four groups $(<1,1-2,3-14, \geq 15)$ and attack duration in hours (D) into three $(<4,4-12,>12)$. Because $\mathrm{F}$ reflected days with headache rather than attack frequency, we used a conservative approximation method for calculation of proportion of time in ictal state (pT). For participants reporting $\mathrm{D} \leq 24 \mathrm{~h}, \mathrm{pT}$ was calculated as $\left[\left(\mathrm{F}^{*} \mathrm{D}\right) /(30 * 24)\right]$. When $\mathrm{D}>24 \mathrm{~h}$, we assumed $\mathrm{F}$ was accordingly inflated (for example, one attack lasting into 2 days was reported as 2 headache days); we therefore calculated $\mathrm{pT}$ as $\left[\left(\mathrm{F}^{*} 24\right) /\left(30^{*} 24\right)\right]=\mathrm{F} / 30$. We converted proportions into percentages, and categorized these into four groups $(<1 \%, 1-2 \%, 2.1-8 \%,>8 \%)$.

We used chi-squared to test differences in proportions reporting medical consultation(s), investigations and/or treatments, according to headache type, habitation and/ or dwelling altitude. We used bivariate and multivariate logistic regression analyses (with odds ratios [ORs] and adjusted ORs [AORs], each with 95\% CIs) to investigate associations of medical consultation with demographic variables and indices of symptom severity. We entered each of these as covariates in the multivariate analyses, but only the demographic variables for associations with symptom severity. We considered $p<0.05$ to be statistically significant.

We analysed all data using SPSS 21.0 (IBM Corp, Armonk, NY, USA).

\section{Results}

A total of 2100 participants were included in the original survey, but here we analyze only the 1794 who reported headache during the preceding year (mean age $36.1 \pm \mathrm{SD}$ 12.6 years; $\mathrm{M} / \mathrm{F}$ ratio $1: 1.6 ; 38.2 \%$ from poorest category; $38.2 \%$ rural; $52.9 \%$ dwelling at altitude $\geq 1000 \mathrm{~m}$ ).

\section{Medical consultation}

Of the 1794 participants reporting headache, 1048 (58.4\%; 95\% CI: 56.1-60.7\%) consulted at some level within the preceding year for headache, $25.0 \%(\mathrm{M} / \mathrm{F}$ ratio 1:1.1) with paramedics, $15.0 \%$ (M/F ratio 1:0.96]) with pharmacists, $10.8 \%(\mathrm{M} / \mathrm{F}$ ratio $1: 1.1)$ with general physicians and 7.6\% (M/F ratio: 1:1.4) with specialists (Fig. 1). By headache type, participants with $\mathrm{pMOH}(87.0 \%)$ were most likely to have consulted, followed by those with migraine $(67.2 \%)$, then those with TTH $(48.6 \% ; p<0.001)$ (Table 1).

\section{Investigations and treatments}

Of the 1794 participants reporting headache, rather more than one tenth $(11.9 \%)$ were investigated for headache within the preceding year, most commonly $(8.9 \%)$ by eye test (Table 2$)$. Next, but far fewer, were xrays of PNS (1.4\%); brain imaging $(0.9 \%)$ was unusual, and neck xrays (0.4\%) and EEG (0.3\%) even more so (Table 2).

Just over half $(50.8 \%)$ had used at least one type of conventional medication for headache in the preceding month. Among 13 different medications (paracetamol, aspirin, diclofenac, ibuprofen, naproxen, nimesulide,

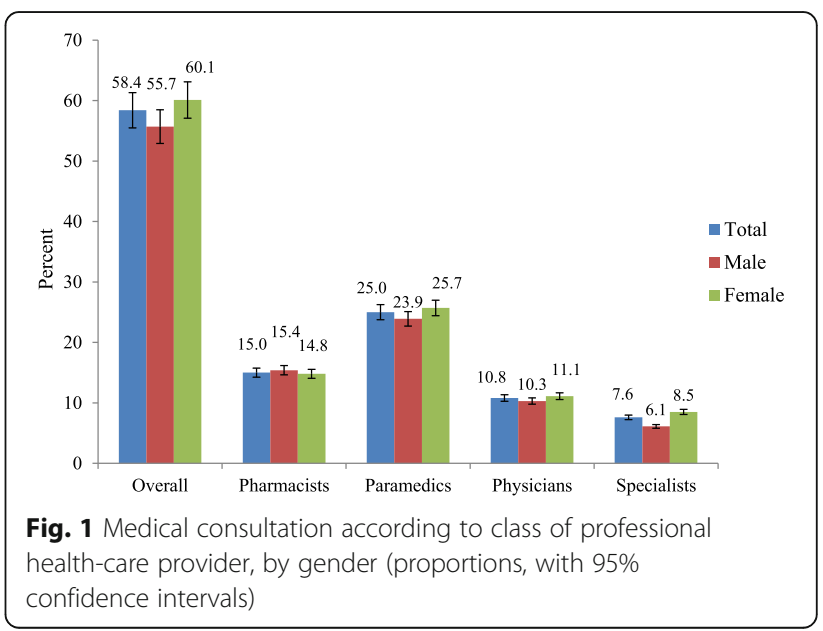


Table 1 Medical consultation according to class of professional health-care provider, by headache type

\begin{tabular}{|c|c|c|c|c|c|c|}
\hline \multirow[t]{2}{*}{ Headache type } & \multicolumn{6}{|c|}{ Medical consultation } \\
\hline & \multirow[t]{2}{*}{ N } & Pharmacist & Paramedic & Physician & Specialist & Overall \\
\hline & & \multicolumn{5}{|c|}{ n (\%) [95\% confidence interval] } \\
\hline Tension-type headache & 863 & $\begin{array}{l}123(14.3) \\
{[12.0-16.8]}\end{array}$ & $\begin{array}{l}185(21.4) \\
{[18.7-24.3]}\end{array}$ & $\begin{array}{l}64(7.4) \\
{[5.8-9.4]}\end{array}$ & $\begin{array}{l}47(5.4) \\
{[4.0-7.2]}\end{array}$ & $\begin{array}{l}419(48.6) \\
{[45.2-51.9]}\end{array}$ \\
\hline Migraine & 728 & $\begin{array}{l}117(16.1) \\
{[13.5-18.9]}\end{array}$ & $\begin{array}{l}216(29.7) \\
{[26.4-33.1]}\end{array}$ & $\begin{array}{l}101(13.9) \\
{[11.4-16.6]}\end{array}$ & $\begin{array}{l}55(7.6) \\
{[5.7-9.7]}\end{array}$ & $\begin{array}{l}489(67.2) \\
{[63.6-70.6]}\end{array}$ \\
\hline Probable medication-overuse headache & 46 & $\begin{array}{l}9(19.6) \\
{[9.4-33.9]}\end{array}$ & $\begin{array}{l}10(21.7) \\
{[10.9-36.4]}\end{array}$ & $\begin{array}{l}11(23.9) \\
{[12.6-38.8]}\end{array}$ & $\begin{array}{l}10(21.7) \\
{[10.9-36.4]}\end{array}$ & $\begin{array}{l}40(87.0) \\
{[73.7-95.6]}\end{array}$ \\
\hline$p^{*}$ & - & $<0.001$ & $<0.001$ & $<0.001$ & $<0.001$ & $<0.001$ \\
\hline
\end{tabular}

$\mathrm{p}^{*}$ : chi-squared test

Table 2 Investigations and treatments for headache of participants with any headache, by habitation and dwelling altitude

\begin{tabular}{|c|c|c|c|c|c|c|c|}
\hline & \multirow[t]{2}{*}{ All } & \multicolumn{3}{|l|}{ Habitation } & \multicolumn{3}{|l|}{ Dwelling altitude } \\
\hline & & Urban & Rural & $p^{*}$ & $<1000 \mathrm{~m}$ & $\geq 1000 \mathrm{~m}$ & $p^{*}$ \\
\hline & \multicolumn{4}{|c|}{$\mathrm{n}(\%)[95 \% \mathrm{Cl}]$} & \multicolumn{3}{|l|}{$\mathrm{n}(\%)[95 \% \mathrm{Cl}]$} \\
\hline \multicolumn{8}{|l|}{ Investigations in the preceding year } \\
\hline All & $\begin{array}{l}214(11.9) \\
{[10.5-13.5]}\end{array}$ & $\begin{array}{l}75(10.9) \\
{[8.7-13.5]}\end{array}$ & $\begin{array}{l}139(12.5) \\
{[10.7-14.6]}\end{array}$ & 0.33 & $\begin{array}{l}106(12.5) \\
{[10.4-15.0]}\end{array}$ & $\begin{array}{l}108(11.4) \\
{[9.4-13.6]}\end{array}$ & 0.47 \\
\hline Xray of paranasal sinuses & $\begin{array}{l}26(1.4) \\
{[0.9-2.1]}\end{array}$ & $\begin{array}{l}5(0.7) \\
{[0.2-1.7]}\end{array}$ & $\begin{array}{l}21(1.9) \\
{[1.2-2.9]}\end{array}$ & 0.07 & $\begin{array}{l}12(1.4) \\
{[0.7-2.5]}\end{array}$ & $\begin{array}{l}14(1.5) \\
{[0.8-2.5]}\end{array}$ & 0.99 \\
\hline Xray of neck & $\begin{array}{l}7(0.4) \\
{[0.2-0.6]}\end{array}$ & $\begin{array}{l}3(0.4) \\
{[0.1-1.3]}\end{array}$ & $\begin{array}{l}4(0.4) \\
{[0.1-0.9]}\end{array}$ & 1.0 & $\begin{array}{l}1(0.1) \\
{[0.0-0.7]}\end{array}$ & $\begin{array}{l}6(0.6) \\
{[0.2-1.4]}\end{array}$ & 0.13 \\
\hline Brain imaging (CT or MRI) & $\begin{array}{l}16(0.9) \\
{[0.5-0.9]}\end{array}$ & $\begin{array}{l}7(1.0) \\
{[0.4-2.1]}\end{array}$ & $\begin{array}{l}9(0.8) \\
{[0.4-1.5]}\end{array}$ & 0.79 & $\begin{array}{l}9(1.1) \\
{[0.5-2.0]}\end{array}$ & $\begin{array}{l}7(0.7) \\
{[0.3-1.5]}\end{array}$ & 0.61 \\
\hline EEG & $\begin{array}{l}5(0.3) \\
{[0.1-0.5]}\end{array}$ & $\begin{array}{l}1(0.1) \\
{[0.0-0.8]}\end{array}$ & $\begin{array}{l}4(0.4) \\
{[0.1-0.9]}\end{array}$ & 0.65 & $\begin{array}{l}0(0.0) \\
-\end{array}$ & $\begin{array}{l}5(0.5) \\
{[0.2-1.2]}\end{array}$ & - \\
\hline Eye test & $\begin{array}{l}160(8.9) \\
{[7.6-10.3]}\end{array}$ & $\begin{array}{l}59(8.6) \\
{[6.6-11.0]}\end{array}$ & $\begin{array}{l}101(9.1) \\
{[7.5-11.0]}\end{array}$ & 0.73 & $\begin{array}{l}82(9.7) \\
{[7.8-11.9]}\end{array}$ & $\begin{array}{l}78(8.2) \\
{[6.6-10.2]}\end{array}$ & 0.28 \\
\hline \multicolumn{8}{|c|}{ Conventional medications in the preceding month } \\
\hline Overall & $\begin{array}{l}912(50.8) \\
{[48.5-53.2]}\end{array}$ & $\begin{array}{l}299(43.6) \\
{[39.8-47.4]}\end{array}$ & $\begin{array}{l}613(55.3) \\
{[52.3-58.3]}\end{array}$ & $<0.001$ & $393(46.5)[43.1-49.9]$ & $\begin{array}{l}519(54.7) \\
{[51.5-57.9]}\end{array}$ & $<0.001$ \\
\hline Paracetamol & $\begin{array}{l}682(38.0) \\
{[35.8-40.3]}\end{array}$ & $\begin{array}{l}203(29.6) \\
{[26.2-33.2]}\end{array}$ & $\begin{array}{l}479(43.2) \\
{[41.9-47.8]}\end{array}$ & $<0.001$ & $\begin{array}{l}250(29.6) \\
{[26.5-32.8]}\end{array}$ & $\begin{array}{l}432(45.5) \\
{[42.3-48.8]}\end{array}$ & $<0.001$ \\
\hline Ibuprofen & $\begin{array}{l}150(8.4) \\
{[7.1-9.7]}\end{array}$ & $\begin{array}{l}57(8.3) \\
{[6.4-10.6]}\end{array}$ & $\begin{array}{l}93(8.4) \\
{[6.8-10.2]}\end{array}$ & 0.99 & $\begin{array}{l}51(5.9) \\
{[4.5-7.9]}\end{array}$ & $\begin{array}{l}99(10.4) \\
{[8.6-12.6]}\end{array}$ & 0.001 \\
\hline Nimesulide & $\begin{array}{l}94(5.2) \\
{[4.3-6.4]}\end{array}$ & $\begin{array}{l}49(7.1) \\
{[5.3-9.3]}\end{array}$ & $\begin{array}{l}45(4.1) \\
{[3.0-5.4]}\end{array}$ & 0.009 & $\begin{array}{l}68(8.0) \\
{[6.3-10.1]}\end{array}$ & $\begin{array}{l}26(2.7) \\
{[1.8-4.0]}\end{array}$ & $<0.001$ \\
\hline NSAID/paracetamol combination & $\begin{array}{l}71(4.0) \\
{[3.1-5.0]}\end{array}$ & $\begin{array}{l}18(2.6) \\
{[1.6-4.1]}\end{array}$ & $\begin{array}{l}53(4.8) \\
{[3.6-6.2]}\end{array}$ & 0.025 & $\begin{array}{l}43(5.1) \\
{[3.7-6.8]}\end{array}$ & $\begin{array}{l}28(3.0) \\
{[2.0-4.1]}\end{array}$ & 0.021 \\
\hline \multicolumn{8}{|c|}{ Herbal therapies in the preceding month } \\
\hline Overall & $\begin{array}{l}185(10.3) \\
{[8.9-11.8]}\end{array}$ & $\begin{array}{l}36(5.2) \\
{[3.7-7.2]}\end{array}$ & $\begin{array}{l}149(13.4) \\
{[11.5-15.6]}\end{array}$ & $<0.001$ & $\begin{array}{l}63(7.5) \\
{[5.8-9.4]}\end{array}$ & $122(12.9)[10.8-15.2]$ & $<0.001$ \\
\hline Vicks & $\begin{array}{l}64(3.6) \\
{[2.8-4.6]}\end{array}$ & $\begin{array}{l}15(2.2) \\
{[1.2-3.6]}\end{array}$ & $\begin{array}{l}49(4.4) \\
{[3.3-5.8]}\end{array}$ & 0.013 & $\begin{array}{l}36(4.3) \\
{[3.0-5.8]}\end{array}$ & $\begin{array}{l}28(3.0) \\
{[3.0-4.3]}\end{array}$ & 0.16 \\
\hline Tulsi leaves & $\begin{array}{l}11(0.6) \\
{[0.3-1.1]}\end{array}$ & $\begin{array}{l}3(0.4) \\
{[0.1-1.3]}\end{array}$ & $\begin{array}{l}8(0.7) \\
(0.3-1.4)\end{array}$ & 0.55 & $\begin{array}{l}7(0.8) \\
{[0.3-1.7]}\end{array}$ & $\begin{array}{l}4(0.4) \\
{[0.1-1.1]}\end{array}$ & 0.37 \\
\hline Titepati leaves & $\begin{array}{l}11(0.6) \\
{[0.3-1.1]}\end{array}$ & $\begin{array}{l}3(0.4) \\
{[0.1-1.3]}\end{array}$ & $\begin{array}{l}8(0.7) \\
{[0.3-1.4]}\end{array}$ & 0.55 & $\begin{array}{l}2(0.2) \\
{[0.0-0.9]}\end{array}$ & $\begin{array}{l}9(0.9) \\
{[0.4-1.8]}\end{array}$ & 0.069 \\
\hline
\end{tabular}

Cl Confidence interval

$\mathrm{p}^{*}$ : chi-squared test

Significant $p$-values $(<0.05)$ are emboldened 
codeine or dihydrocodeine, domperidone, metoclopramide, Codamol, Coflam, D-cold and Rhinex), the most common was paracetamol as monotherapy (38.0\%); ibuprofen (8.4\%) followed way behind. Nimesulide was used by a not inconsiderable minority (5.2\%). Less common was the combination of nonsteroidal anti-inflammatory drug (NSAID) and paracetamol (4.0\%) (Table 2).

Just over one tenth (10.3\%) had used herbal therapies in the last month, most commonly Vicks (3.6\%). This was usually applied to the forehead, inhaled or administered via the nasal mucosa. Depending on formulation, it might contain camphor, turpentine oil, levomenthol, eucalyptus oil, Siberian pine needle oil, methyl salicylate and/or oxymetazoline hydrochloride. Next, but not common, were tulsi leaves (Holy basil, or Ocimum tenuiflorum) and titepati leaves (Artemesia vulgaris) (both $0.6 \%$ ) (Table 2). These were usually taken as a drink after boiling in water.

\section{Associations}

Overall, medical consultations were similar in females $(60.1 \%)$ and males (55.7\%; $p=0.96)$ (Fig. 1, Tables 3 and 4). Females (8.5\%) consulted specialists more than males (6.1\%), but not significantly ( $p=0.28$ ) (Tables 3 and 4 ).

There was a slightly increasing overall trend with advancing age up to $46-55$ years (from $56.3 \%$ for

Table 3 Medical consultation by age, gender, household consumption, habitation and dwelling altitude

\begin{tabular}{|c|c|c|c|c|c|}
\hline \multirow[t]{2}{*}{ Variable } & \multirow[t]{2}{*}{ N } & \multicolumn{2}{|l|}{ Overall } & \multicolumn{2}{|c|}{ Specialist } \\
\hline & & n (\%) & $95 \% \mathrm{Cl}$ & n (\%) & $95 \% \mathrm{Cl}$ \\
\hline \multicolumn{6}{|l|}{ Age (years) } \\
\hline $18-25$ & 426 & $240(56.3)$ & $51.5-61.1$ & $24(5.6)$ & $3.6-8.3$ \\
\hline $26-35$ & 581 & $346(59.6)$ & $55.4-63.6$ & $54(9.3)$ & $7.1-12.0$ \\
\hline $36-45$ & 358 & $204(57.0)$ & $51.7-62.2$ & $25(7.0)$ & $4.6-10.1$ \\
\hline $46-55$ & 258 & $162(62.9)$ & $56.6-68.7$ & $18(7.0)$ & $4.2-10.8$ \\
\hline $56-65$ & 171 & $96(56.1)$ & $48.4-63.7$ & $15(8.8)$ & $5.0-14.1$ \\
\hline \multicolumn{6}{|l|}{ Gender } \\
\hline Male & 689 & $384(55.7)$ & $51.9-59.5$ & $42(6.1)$ & $4.4-8.2$ \\
\hline Female & 1105 & $664(60.1)$ & $57.1-63.0$ & $94(8.5)$ & $6.9-10.3$ \\
\hline \multicolumn{6}{|c|}{ Household consumption (USD/year) } \\
\hline$<950$ & 686 & $423(61.7)$ & $57.9-65.3$ & $46(6.7)$ & $5.0-8.8$ \\
\hline $950-1200$ & 687 & $400(58.2)$ & $54.4-61.9$ & $61(8.9)$ & $6.9-11.3$ \\
\hline$>1200$ & 421 & $225(53.4)$ & $48.6-58.3$ & $29(6.9)$ & $4.7-9.7$ \\
\hline \multicolumn{6}{|l|}{ Habitation } \\
\hline Urban & 686 & 354 (51.6) & $47.8-55.4$ & $54(7.9)$ & $6.0-10.1$ \\
\hline Rural & 1108 & $694(62.6)$ & $59.7-65.5$ & $82(7.4)$ & $5.9-9.1$ \\
\hline \multicolumn{6}{|c|}{ Dwelling altitude (m) } \\
\hline$<1000$ & 845 & $474(56.1)$ & $52.7-59.5$ & $72(8.5)$ & $6.7-10.6$ \\
\hline$\geq 1000$ & 949 & $574(60.5)$ & $57.3-63.6$ & $64(6.7)$ & $5.2-8.5$ \\
\hline
\end{tabular}

$\mathrm{Cl}$ confidence interval
18-25 years to $62.9 \%$ for $46-55$ year), then a decrease (to $56.1 \%$ for $56-65$ years) (Tables 3 and 4 ). However, age was not a significant factor (AOR 1.2 [ $p=0.28]$ for $46-55$ years and AOR 0.8 [ $p=0.28]$ for 56-65 years with reference to the youngest group) (Tables 3 and 4). Consultations with specialists increased with age (5.6\% for $18-25$ years, $9.3 \%$ for $26-35$ years, $7.0 \%$ for $36-55$ years and $8.8 \%$ for $56-65$ years), but again not significantly (Tables 3 and 4 ).

Medical consultation showed no associations with household consumption or dwelling altitude. Rural participants consulted more than urban $(\mathrm{OR}=1.6 ; \mathrm{AOR}=$ 1.5; $p<0.001$ ) (Tables 3 and 4). Specialist consultation showed no demographic associations.

Investigations showed no associations, but both conventional medications and herbal therapies were reportedly used more, overall, by rural than by urban participants $(p<0.001)$, and by high-altitude dwellers than low $(p<0.001)$ (Table 2).

Associations with indices of symptom severity are presented in Table 5. In bivariate analysis, greater severity (according to all indices) was associated with increased likelihood of medical consultation. Multivariate analysis confirmed these associations. This was not the case for specialist consultation. Greater headache frequency showed a paradoxical association, with ORs and AORs of $0.6-0.7$ for frequencies of $1-14 \mathrm{~d} / \mathrm{m}$ but $>2$ for frequency $\geq 15 \mathrm{~d} / \mathrm{m}$. Reflecting this, specialist consultation was less likely initially (OR and $A O R=0.5)$ as $\mathrm{pT}$ increased above $2 \%$, then becoming more likely beyond $\mathrm{pT}=8 \%$. Greater headache intensity was clearly associated with specialist consultation in both analyses, whereas the effect of attack duration did not achieve significance (Table 5).

\section{Discussion}

We found that almost three fifths (58.4\%) of all participants reporting headache had at least one consultation with at least one class of professional HCP in the previous year, while fewer than one-tenth (7.6\%) had seen a specialist of any sort. Unsurprisingly, the great majority (87.0\%) of participants with pMOH had consulted, as had two-thirds (67.2\%) with migraine but barely half (48.7\%) with TTH. In keeping with this, the likelihood of having consulted increased with all indices of symptom severity, although only headache intensity and frequency of $\geq 15 \mathrm{~d} / \mathrm{m}$ showed clear associations with specialist consultation. Over one tenth had been investigated. Half had used conventional medications, and one tenth had used herbal therapies for headache in the preceding month.

It may seem remarkable that such a large proportion of the participants with headache had consulted: this finding, in a low-income country, suggests better 
Table 4 Associations between medical consultation and demographic variables

\begin{tabular}{|c|c|c|c|c|c|c|c|c|}
\hline \multirow[t]{3}{*}{ Variable } & \multicolumn{4}{|l|}{ Overall } & \multicolumn{4}{|c|}{ Specialist consultation } \\
\hline & \multicolumn{2}{|c|}{ Bivariate analysis } & \multicolumn{2}{|c|}{ Multivariate analysis } & \multicolumn{2}{|c|}{ Bivariate analysis } & \multicolumn{2}{|c|}{ Multivariate analysis } \\
\hline & $\overline{\mathrm{OR}}(95 \% \mathrm{Cl})$ & $\mathrm{p}$ & $\mathrm{AOR}^{\mathrm{a}}(95 \% \mathrm{Cl})$ & $\mathrm{p}$ & $\overline{O R}(95 \% \mathrm{Cl})$ & $p$ & $\mathrm{AOR}^{\mathrm{a}}(95 \% \mathrm{Cl})$ & $p$ \\
\hline \multicolumn{9}{|l|}{ Age (in years) } \\
\hline $18-25$ & Reference & - & Reference & - & Reference & - & Reference & - \\
\hline $26-35$ & $1.1(0.9-1.5)$ & 0.31 & $1.1(0.8-1.4)$ & 0.54 & $1.7(1.1-2.8)$ & 0.034 & $1.5(0.9-2.5)$ & 0.11 \\
\hline $36-45$ & $1.0(0.8-1.4)$ & 0.86 & $0.9(0.7-1.3)$ & 0.62 & $1.3(0.7-2.4)$ & 0.44 & $1.2(0.6-2.1)$ & 0.65 \\
\hline $46-55$ & $1.3(0.6-1.8)$ & 0.10 & $1.2(0.9-1.7)$ & 0.28 & $1.3(0.7-2.4)$ & 0.48 & $1.1(0.6-2.2)$ & 0.71 \\
\hline $56-65$ & $1.0(0.7-1.5)$ & 0.96 & $0.8(0.6-1.2)$ & 0.28 & $1.6(0.8-3.2)$ & 0.16 & $1.5(0.8-3.0)$ & 0.24 \\
\hline \multicolumn{9}{|l|}{ Gender } \\
\hline Male & Reference & - & Reference & - & Reference & - & Reference & - \\
\hline Female & $1.2(0.9-1.5)$ & 0.069 & $1.0(0.8-1.2)$ & 0.96 & $1.4(1.0-2.1)$ & 0.062 & $1.2(0.8-1.8)$ & 0.28 \\
\hline \multicolumn{9}{|c|}{ Household consumption (USD/year) } \\
\hline$<950$ & $1.2(0.9-1.4)$ & 0.19 & $1.1(0.9-1.4)$ & 0.40 & $0.7(0.5-1.1)$ & 0.13 & $0.8(0.5-1.1)$ & 0.17 \\
\hline $950-1200$ & Reference & - & Reference & - & Reference & - & Reference & - \\
\hline$>1200$ & $0.8(0.6-1.1)$ & 0.12 & $0.8(0.6-1.0)$ & 0.11 & $0.8(0.5-1.2)$ & 0.24 & $0.7(0.5-1.2)$ & 0.20 \\
\hline \multicolumn{9}{|l|}{ Habitation } \\
\hline Urban & Reference & - & Reference & - & Reference & - & Reference & - \\
\hline Rural & $1.6(1.3-1.9)$ & $<0.001$ & $1.5(1.2-1.9)$ & $<0.001$ & $0.9(0.7-1.3)$ & 0.71 & $0.9(0.6-1.3)$ & 0.51 \\
\hline \multicolumn{9}{|c|}{ Dwelling altitude(m) } \\
\hline$<1000$ & Reference & - & Reference & - & Reference & - & Reference & - \\
\hline$\geq 1000$ & $1.2(0.9-1.4)$ & 0.06 & $1.0(0.8-1.2)$ & 0.96 & $0.8(0.6-1.1)$ & 0.16 & $0.7(0.5-1.1)$ & 0.094 \\
\hline
\end{tabular}

$O R$ odds ratio, $\mathrm{Cl}$ confidence interval, $A O R$ adjusted $O R$

a adjusted for age, gender, household consumption, habitation, dwelling altitude, headache frequency, attack duration, headache intensity and proportion of time in ictal state

Significant $p$-values $(<0.05)$ are emboldened

availability of health care than in many other, wealthier countries in Asia [23, 24], Western Europe [25-27] and North America $[28,29]$. We need to make two cautionary comments on this. First, the evidence from other countries came from studies conducted some two decades earlier [24-29]. In that period, more effective treatments for headache have emerged, awareness of headache as a major public-health concern has substantially increased [30, 31], and, of course, information technology has advanced greatly. These may have led to changes in policy priorities and in public health-seeking behaviour. Second, and probably more influential, we included consultations with a very wide range of HCPs in our count of "medical consultations" in Nepal, among them some who have no counterparts in many other countries, or would not be accredited as health professionals. This probably lies behind the otherwise surprising finding that medical consultation was positively associated with rural habitation (discussed below). If pharmacist consultations (15.0\%) are excluded, as they might be in studies elsewhere, the medical consultation proportion falls to $43.4 \%$ in Nepal, similar to the $46.6 \%$ recently found in China [32] and somewhat lower than findings in other countries [23-29]. If consultations only with physicians (general physicians [10.8\%] or specialists [7.6\%]) are considered, the proportion in Nepal (18.4\%) is much lower than those elsewhere [23-29, 32]. This is probably the most salient comparison. It should be added that, while we included a range of specialties in the "specialist" classification (allowing for the possibility of consultations abroad), Nepal has no headache specialists, and few neurologists or neurosurgeons, so consultations were most likely with ophthalmologists, ENT specialists or psychiatrists.

In other words, these findings should not be taken as indicators of good care. The high consultation proportion reflects high demand, but gives no assurance that needs were met. On the contrary, headache-attributed burden in Nepal persists at a high level [16]. Investigations were requested for over $10 \%$, but most were eye (vision) tests. While these might have been clinically indicated, they would not have contributed much to headache diagnosis or management [21]. Among the medications used, paracetamol was prominent - far more than NSAIDs, despite being less effective [33]. Nimesulide, a drug giving rise to serious safety concerns [34], was reportedly used by over $5 \%$. These are clear markers of health-care failure. 
Table 5 Associations between medical consultation and indices of symptom severity

\begin{tabular}{|c|c|c|c|c|c|c|c|c|}
\hline \multirow[t]{3}{*}{ Index } & \multicolumn{4}{|l|}{ Overall } & \multicolumn{4}{|c|}{$\underline{\text { Specialist consultation }}$} \\
\hline & \multicolumn{2}{|c|}{ Bivariate analysis } & \multicolumn{2}{|c|}{ Multivariate analysis } & \multicolumn{2}{|c|}{ Bivariate analysis } & \multicolumn{2}{|c|}{ Multivariate analysis } \\
\hline & OR (95\% Cl) & $p$ & $\mathrm{AOR}^{\mathrm{a}}(95 \% \mathrm{Cl})$ & $p$ & OR $(95 \% \mathrm{Cl})$ & $p$ & $\mathrm{AOR}^{\mathrm{a}}(95 \% \mathrm{Cl})$ & $p$ \\
\hline \multicolumn{9}{|c|}{ Headache frequency $(\mathrm{d} / \mathrm{m})$} \\
\hline$<1$ & Reference & - & Reference & - & Reference & - & Reference & - \\
\hline $1-2$ & $1.2(0.9-1.5)$ & 0.18 & $1.2(0.9-1.5)$ & 0.17 & $0.6(0.4-0.9)$ & 0.025 & $0.6(0.4-0.9)$ & 0.023 \\
\hline $3-14$ & $1.8(1.4-2.3)$ & $<0.001$ & $1.9(1.5-2.4)$ & $<0.001$ & $0.7(0.4-1.1)$ & 0.14 & $0.7(0.4-1.1)$ & 0.12 \\
\hline$\geq 15$ & $2.7(1.8-3.9)$ & $<0.001$ & $2.6(1.8-3.9)$ & $<0.001$ & $2.2(1.3-3.6)$ & 0.002 & $2.1(1.3-3.5)$ & 0.004 \\
\hline \multicolumn{9}{|c|}{ Attack duration (hours) } \\
\hline$<4$ & Reference & - & Reference & - & Reference & - & Reference & - \\
\hline $4-12$ & $2.0(1.6-2.6)$ & $<0.001$ & $2.1(1.6-2.6)$ & $<0.001$ & $1.5(0.9-2.3)$ & 0.13 & $1.4(0.9-2.3)$ & 0.14 \\
\hline$>12$ & $3.1(2.5-4.3)$ & $<0.001$ & $3.3(2.3-4.1)$ & $<0.001$ & $1.5(0.9-2.2)$ & 0.087 & $1.4(0.9-2.4)$ & 0.10 \\
\hline \multicolumn{9}{|c|}{ Headache intensity } \\
\hline Mild & Reference & - & Reference & - & Reference & - & Reference & - \\
\hline Moderate & $2.5(2.0-3.2)$ & $<0.001$ & $2.5(2.0-3.2)$ & $<0.001$ & $1.3(0.8-2.3)$ & 0.30 & $1.3(0.8-2.3)$ & 0.31 \\
\hline Severe & $3.8(2.9-5.1)$ & $<0.001$ & $3.6(2.7-4.8)$ & $<0.001$ & $2.6(1.5-4.5)$ & 0.001 & $2.7(1.3-4.7)$ & $<0.001$ \\
\hline \multicolumn{9}{|c|}{ Proportion of time in ictal state (pT) (\%) } \\
\hline$<1$ & Reference & - & Reference & - & Reference & - & Reference & - \\
\hline $1-2$ & $1.5(1.1-2.0)$ & 0.012 & $1.5(1.1-2.0)$ & 0.014 & $1.0(0.6-1.7)$ & 0.92 & $1.0(0.5-1.7)$ & 0.95 \\
\hline $2.1-8$ & $1.6(1.2-2.1)$ & 0.001 & $1.6(1.2-2.1)$ & 0.001 & $0.5(0.2-0.9)$ & 0.023 & $0.5(0.3-0.9)$ & 0.021 \\
\hline$>8$ & $2.8(2.2-3.5)$ & $<0.001$ & $2.9(2.3-3.7)$ & $<0.001$ & $1.4(0.9-2.1)$ & 0.094 & $1.4(0.9-2.0)$ & 0.13 \\
\hline
\end{tabular}

$O R$ odds ratio, $\mathrm{Cl}$ confidence interval, $A O R$ adjusted $\mathrm{OR}$

a adjusted for age, gender, household consumption, habitation and dwelling altitude

Significant $p$-values $(<0.05)$ are emboldened

But a more positive view may be taken. Over half of participants with headache had engaged at some level with professional health care, a proportion well in line with international recommendations for headache service organisation and delivery [31]. This indicates that capacity is available within Nepalese health services, and this can be built upon in a programme aimed at improvement. Furthermore, there is an existing health-care infrastructure that would readily accommodate the suggested three-tier model of headache-service organization [31]. Consultations with pharmacists (whose important role is to keep those with simple requirements outside the three-tier model) might be entirely adequate for the $15.0 \%$ reporting only these - if pharmacists had basic knowledge and skills in management of headache. This proviso applies equally to nurses, health assistants and auxiliary health workers (who would be at level one within the model) and, indeed, general physicians (level two). While only $10.8 \%$ had seen general physicians, and only $7.6 \%$ had seen a specialist of any sort (level three), these should suffice if all HCPs received training in headache care appropriate to their level [31].

Nepal is a low-income country; the range of household consumption (a marker of socio-economic status) is very much compressed towards the lower end. We did not see associations between household consumption and medical consultation, overall or with specialists, but any such relationship would be difficult to detect for this reason. We did observe that rural participants with headache were more likely than their urban counterparts to consult, although not with specialists. This, seen also in both Taiwan [24] and mainland China [32], may be due in Nepal to the provision nationwide but especially in rural areas of easy-access community-based primary health-care centres (PHCs), staffed mainly by health assistants and/or auxiliary health workers [35]. Nepal has a mixed health-service model, involving both public and private sectors. While government-supported basic services are available throughout the country, PHCs offer free medical consultations as well as being easily accessible [35]. Private-sector health care, predominantly available in urban areas, requires payment. Other likely factors are greater rural recourse to herbal practitioners, while a lesser tendency to self-medication in rural areas may drive people to consult health professionals.

As anticipated, all indices of symptom severity were positive predictors of medical consultation. Only headache intensity and high frequency predicted consultation with specialists, but numbers in these analyses were small, while attack duration (also a factor in proportion of time in ictal state) tends to be unreliably reported [19, 22]. A 
point worthy of emphasis here is that these indices of symptom severity are, clearly, key indicators of need for headache services. We have previously shown that all symptom indices increase with altitude of dwelling across the range $<500 \mathrm{~m}$ to $2499 \mathrm{~m}$ [36], and this was reflected here in the greater use of medication by high-altitude dwellers. Clearly, this relative excess of need among high-altitude dwellers presents a major challenge to equitable provision of headache services in Nepal. But need is very high in Nepal $[15,16]$, so it needs to be done.

\section{Strengths and limitations}

The cross-sectional study from which our data were drawn used methods tested earlier in many countries, including India with a not dissimilar culture $[9,10]$. Despite major logistic difficulties [15], it recruited a large, nationally representative sample through careful random selection, and minimized participation bias by achieving a participation proportion of $>99 \%$ [18]. These were strengths of the study.

Two limitations were inherent in population-based surveys. Enquiry into medical consultations was based on participants' recall over the preceding year, with some degree of error expected. We were similarly dependent on participants' truthfulness, although they had no reason to be evasive in this type of enquiry. Any errors that were introduced were more likely to be random than systematic $[32,37]$. A third limitation was that no enquiry was possible into outcomes - in particular, satisfaction with care received as a result of consultation. This would have required much more detailed enquiry, beyond our resources. What is clear, since the burden of headache remains very high [16], is that needs in Nepal are largely unmet, despite the high proportion consulting HCPs.

\section{Conclusions}

While more than half of participants with headache had consulted a professional HCP within the previous year, this statistic reflects demand while saying nothing of the quality of care given. Neither the investigations reported nor the medications used offer reassurance, while high persistent burden more plainly indicates these consultations fall far short of meeting need. Health policy in Nepal would do well to recognise this: the consequences otherwise are costly - in lost health, in diminished productivity, and to the national economy. Importantly, capacity appears to exist at multiple levels within the Nepalese health system. With this to build upon, structured headache services in line with international recommendations appear to be achievable in Nepal, despite the obvious difficulties. The essential requirement is educational.

\section{Abbreviations}

AOR: Adjusted odds ratio; Cl: Confidence interval; $C$ : Computed tomography; EEG: Electroencephalography; ENT: Ear, nose and throat; GBD: Global Burden of Disease study; HARDSHIP: Headache-Attributed
Restriction, Disability, Social Handicap and Impaired Participation; HCP: Health-care provider; ICHD: International Classification of Headache Disorders; LAMI: Low- and middle-income; LTB: Lifting The Burden; $\mathrm{MOH}$ : Medication-overuse headache; MRI: Magnetic resonance imaging; NPR: Nepalese rupee; NSAID: Nonsteroidal anti-inflammatory drug; OR: Odds ratio; PHC: Primary health-care centre; $\mathrm{PMOH}$ : Probable $\mathrm{MOH}$; PNS: Paranasal sinuses; SD: Standard deviation; SEAR: South-East Asia Region; SPSS: Statistical Package for Social Sciences; TTH: Tension-type headache; USD: United States dollar; YLD: Year of life lost to disability

\section{Acknowledgements}

This study was performed as a project within the Global Campaign against Headache, which is led by the non-governmental organization Lifting The Burden in official relations with the World Health Organization, and with the support of Dhulikhel Hospital, Kathmandu University Hospital, Dhulikhel, Kavre, Nepal.

\section{Funding}

This study was funded by grants from Samarbeidsorganet, the Liaison Committee between the Central Norway Regional Health Authority and the Norwegian University of Science and Technology (NTNU).

\section{Availability of data and materials}

The data are held on file at Norwegian University of Science and Technology. Once analysis and publications are completed, they will, in line with Global Campaign policy, be freely available for non-commercial purposes to any person requesting access.

\section{Authors' contributions}

All authors were involved in conception and design of the study. AR and KM acquired data with support from ML and TJS. KM and TJS engaged in data analysis and interpretation. KM drafted the manuscript. All authors revised it critically for intellectual content, and approved the version submitted.

\section{Ethics approval and consent to participate}

The study protocol was approved by the Nepal Health Research Council, the Institutional Review Committee of Kathmandu University School of Medical Sciences, Dhulikhel Hospital, and the Regional Committee for Health and Research Ethics in Central Norway.

All participants were informed of the nature and purpose of the study, and gave consent, recorded either by signature or by fingerprint in accordance with the requirements of these three committees.

\section{Consent for publication}

Not applicable.

\section{Competing interests}

TJS is a Director and Trustee of Lifting The Burden. Otherwise, the authors declare that they have no competing interests.

\section{Publisher's Note}

Springer Nature remains neutral with regard to jurisdictional claims in published maps and institutional affiliations.

\section{Author details}

${ }^{1}$ Dhulikhel Hospital, Kathmandu University Hospital, Dhulikhel, Kavre, Nepal. ${ }^{2}$ Kathmandu University School of Medical Sciences, Dhulikhel, Kavre, Nepal. ${ }^{3}$ Department of Neuromedicine and Movement Science, Norwegian University of Science and Technology, Edvard Griegs Gate, NO-7489 Trondheim, Norway. ${ }^{4}$ Norwegian Advisory Unit on Headache, St Olavs University Hospital, Trondheim, Norway. ${ }^{5}$ Division of Brain Sciences, Imperial College London, London, UK.

Received: 20 September 2018 Accepted: 4 November 2018

Published online: 28 November 2018

\section{References}

1. World Health Organization and Lifting The Burden (2011) Atlas of headache disorders and resources in the world 2011. WHO, Geneva

2. Jensen R, Stovner $L J$ (2008) Epidemiology and comorbidity of headache. Lancet Neurol 7(4):354-361. https://doi.org/10.1016/s1474-4422(08)70062-0 
3. Linde M, Gustavsson A, Stovner LJ, Steiner TJ, Barre J, Katsarava Z, Lainez JM, Lampl C, Lanteri-Minet M, Rastenyte D, Ruiz de la Torre E, Tassorelli C, Andree C (2012) The cost of headache disorders in Europe: the Eurolight project. Eur J Neurol 19(5):703-711. https://doi.org/10.1111/j.1468-1331.2011. 03612.x

4. Westergaard ML, Hansen EH, Glumer C, Olesen J, Jensen RH (2014) Definitions of medication-overuse headache in population-based studies and their implications on prevalence estimates: a systematic review. Cephalalgia 34(6):409-425. https://doi.org/10.1177/0333102413512033

5. Vos T, Allen C, Arora M, Barber RM, Bhutta ZA, Brown A, Carter A, Casey DC, Charlson FJ, Chen AZ, Coggeshall M, Cornaby L, Dandona L, Dicker DJ, Dilegge T, Erskine HE, Ferrari AJ, Fitzmaurice C, Fleming T, Forouzanfar $\mathrm{MH}$, Fullman N, Gething PW, Goldberg EM, Graetz N, Haagsma JA, Johnson CO, Kassebaum NJ et al (2016) Global, regional, and national incidence, prevalence, and years lived with disability for 310 diseases and injuries, 1990-2015: a systematic analysis for the global burden of disease study 2015. Lancet 388(10053):1545-1602. https://doi.org/10.1016/s01406736(16)31678-6

6. Vos T, Abajobir AA, Abbafati C, Abbas KM, Abate KH, Abd-Allah F, Abdulle AM, Abebo TA, Abera SF, Aboyans V, Abu-Raddad LJ, Ackerman IN, Adamu AA, Adetokunboh O, Afarideh M, Afshin A, Agarwal SK, Aggarwal R, Agrawal A, Agrawal S, Ahmad Kiadaliri A, Ahmadieh H, Ahmed MB, Aichour AN, Aichour I, Aichour MTE, Aiyar S, Akinyemi RO et al (2017) Global, regional, and national incidence, prevalence, and years lived with disability for 328 diseases and injuries for 195 countries, 1990-2016: a systematic analysis for the global burden of disease study 2016. Lancet (London, England) 390(10100):1211-1259. https://doi.org/10.1016/s0140-6736(17)32154-2

7. Zebenigus M, Tekle-Haimanot R, Worku DK, Thomas H, Steiner TJ (2016) The prevalence of primary headache disorders in Ethiopia. J Headache Pain 17(1):110. https://doi.org/10.1186/s10194-016-0704-z

8. Zebenigus M, Tekle-Haimanot R, Worku DK, Thomas H, Steiner TJ (2017) The burden of headache disorders in Ethiopia: national estimates from a population-based door-to-door survey. J Headache Pain 18(1):58. https://doi. org/10.1186/s10194-017-0765-7

9. Kulkarni GB, Rao GN, Gururaj G, Stovner LJ, Steiner TJ (2015) Headache disorders and public ill-health in India: prevalence estimates in Karnataka state. J Headache Pain 16:67. https://doi.org/10.1186/s10194-015-0549-x

10. Rao GN, Kulkarni GB, Gururaj G, Stovner LJ, Steiner TJ (2015) The burden attributable to headache disorders in India: estimates from a communitybased study in Karnataka state. J Headache Pain 16:94. https://doi.org/10. 1186/s10194-015-0574-9

11. Herekar AA, Ahmad A, Uqaili UL, Ahmed B, Effendi J, Alvi SZ, Shahab MA, Javed U, Herekar AD, Khanani R, Steiner TJ (2017) Primary headache disorders in the adult general population of Pakistan - a cross sectional nationwide prevalence survey. J Headache Pain 18(1):28. https://doi.org/10. 1186/s10194-017-0734-1

12. Mbewe E, Zairemthiama P, Yeh HH, Paul R, Birbeck GL, Steiner TJ (2015) The epidemiology of primary headache disorders in Zambia: a population-based door-to-door survey. J Headache Pain 16:515. https://doi.org/10.1186/ s10194-015-0515-7

13. Mbewe E, Zairemthiama P, Paul R, Birbeck GL, Steiner TJ (2015) The burden of primary headache disorders in Zambia: national estimates from a population-based door-to-door survey. J Headache Pain 16:513. https://doi. org/10.1186/s10194-015-0513-9

14. United States Central Intelligence Agency The World Factbook. https:// www.cia.gov/library/publications/the-world-factbook/geos/np.html. Accessed 12 Mar 2018

15. Manandhar K, Risal A, Steiner TJ, Holen A, Linde M (2015) The prevalence of primary headache disorders in Nepal: a nationwide population-based study. J Headache Pain 16:95. https://doi.org/10.1186/s10194-015-0580-y

16. Manandhar K, Risal A, Linde M, Steiner TJ (2015) The burden of headache disorders in Nepal: estimates from a population-based survey. J Headache Pain 17:3. https://doi.org/10.1186/s10194-016-0594-0

17. Risal A, Manandhar K, Steiner TJ, Holen A, Koju R, Linde M (2014) Estimating prevalence and burden of major disorders of the brain in Nepal: cultural, geographic, logistic and philosophical issues of methodology. J Headache Pain 15:51. https://doi.org/10.1186/1129-2377-15-51

18. Manandhar K, Risal A, Steiner TJ, Holen A, Koju R, Linde M (2014) Estimating the prevalence and burden of major disorders of the brain in Nepal: methodology of a nationwide population-based study. J Headache Pain 15: 52. https://doi.org/10.1186/1129-2377-15-52
19. Steiner TJ, Gururaj G, Andree C, Katsarava Z, Ayzenberg I, Yu SY, Al Jumah M, Tekle-Haimanot R, Birbeck GL, Herekar A, Linde M, Mbewe E, Manandhar K, Risal A, Jensen R, Queiroz LP, Scher Al, Wang SJ, Stovner LJ (2014) Diagnosis, prevalence estimation and burden measurement in population surveys of headache: presenting the HARDSHIP questionnaire. J Headache Pain 15:3. https://doi.org/10.1186/1129-2377-15-3

20. Peters M, Bertolote JM, Houchin C, Kandoura T, Steiner TJ (2007) Tranlation protocol. J Headache Pain 8(Suppl 1):S40-\$47

21. Headache Classification Committee of the International Headache Society (IHS) (2013) The International Classification of Headache Disorders, 3rd edition (beta version). Cephalalgia 33(9):629-808. https://doi.org/10.1177/ 0333102413485658

22. Stovner LJ, Al Jumah M, Birbeck GL, Gururaj G, Jensen R, Katsarava Z, Queiroz LP, Scher Al, Tekle-Haimanot R, Wang SJ, Steiner TJ (2014) The methodology of population surveys of headache prevalence, burden and cost: principles and recommendations from the global campaign against headache. J Headache Pain 15:5. https://doi.org/10.1186/1129-2377-15-5

23. Takeshima T, Ishizaki K, Fukuhara Y, ljiri T, Kusumi M, Wakutani Y, Mori M, Kawashima M, Kowa H, Adachi Y, Urakami K, Nakashima K (2004) Population-based door-to-door survey of migraine in Japan: the Daisen study. Headache 44(1):8-19. https://doi.org/10.1111/j.1526-4610.2004.04004.x

24. Wang SJ, Fuh JL, Young YH, Lu SR, Shia BC (2001) Frequency and predictors of physician consultations for headache. Cephalalgia 21(1):25-30. https://doi. org/10.1046/j.1468-2982.2001.00138.x

25. Rasmussen BK, Jensen R, Olesen J (1992) Impact of headache on sickness absence and utilisation of medical services: a Danish population study. J Epidemiol Community Health 46(4):443-446

26. Lipton RB, Scher Al, Steiner TJ, Bigal ME, Kolodner K, Liberman JN, Stewart WF (2003) Patterns of health care utilization for migraine in England and in the United States. Neurology 60(3):441-448

27. Lampl C, Buzath A, Baumhackl U, Klingler D (2003) One-year prevalence of migraine in Austria: a nation-wide survey. Cephalalgia 23(4):280-286. https:// doi.org/10.1046/j.1468-2982.2003.00509.x

28. Lipton RB, Scher Al, Kolodner K, Liberman J, Steiner TJ, Stewart WF (2002) Migraine in the United States: epidemiology and patterns of health care use. Neurology 58(6):885-894

29. Edmeads J, Findlay H, Tugwell P, Pryse-Phillips W, Nelson RF, Murray TJ (1993) Impact of migraine and tension-type headache on life-style, consulting behaviour, and medication use: a Canadian population survey. Can J Neurol Sci 20(2):131-137

30. Steiner TJ (2005) Lifting The Burden: the global campaign to reduce the burden of headache worldwide. J Headache Pain 6(5):373-377. https://doi. org/10.1007/s10194-005-0241-7

31. Steiner TJ, Antonaci F, Jensen R, Lainez MJ, Lanteri-Minet M, Valade D (2011) Recommendations for headache service organisation and delivery in Europe. J Headache Pain 12(4):419-426. https://doi.org/10.1007/s10194-0110320-X

32. Liu R, Yu S, He M, Zhao G, Yang $X$, Qiao $X$, Feng J, Fang $Y$, Cao $X$, Steiner TJ (2013) Health-care utilization for primary headache disorders in China: a population-based door-to-door survey. J Headache Pain 14:47. https://doi. org/10.1186/1129-2377-14-47

33. Stephens G, Derry S, Moore RA (2016) Paracetamol (acetaminophen) for acute treatment of episodic tension-type headache in adults. Cochrane Database Syst Rev (6):Cd011889. https://doi.org/10.1002/14651858. CD011889.pub2

34. WHO Pharmaceuticals Newsletter 2002, No. 04. http://apps.who.int/ medicinedocs/en/d/Js4943e/3.1.html. Accessed 12 Aug 2018

35. Government of Nepal, Ministry of Health and Population (MOHP) [Nepal] (2014) National Health Policy-2071.

36. Linde M, Edvinsson L, Manandhar K, Risal A, Steiner TJ (2017) Migraine associated with altitude: results from a population-based study in Nepal. Eur J Neurol 24(8):1055-1061. https://doi.org/10.1111/ene.13334

37. Katsarava Z, Mania M, Lampl C, Herberhold J, Steiner TJ (2018) Poor medical care for people with migraine in Europe - evidence from the Eurolight study. J Headache Pain 19(1):10. https://doi.org/10.1186/s10194-018-0839-1 